\title{
Simple and low cost dithizone-functionalized polymer membranes as a tool for mercury preconcentration and monitoring in aqueous bodies. Preliminary results using X-ray absorption near-edge structure (XANES)
}

\author{
Kallithrakas-Kontos N. ${ }^{1,}{ }^{*}$ and Foteinis S. ${ }^{2}$ \\ ${ }^{1}$ Technical University of Crete, Laboratory of Analytical and Environmental Chemistry, University Campus, GR-73100 Chania, Greece \\ 2Public Power Corporation (PPC) Renewables S.A., Attica, Greece \\ Received: 13/08/2018, Accepted: 18/03/2019, Available online: 19/03/2019 \\ *to whom all correspondence should be addressed: e-mail: kalli@mred.tuc.g
} https://doi.org/10.30955/gni.002864

\section{Abstract}

Here the effectiveness of cation selective membranes, produced for divalent mercury $\left(\mathrm{Hg}^{2+}\right)$ preconcetration from aqueous bodies, is examined. To this end, the behavior of PVC-based membranes, functionalized with dithizone for mercury complexation, is examined by means of Energy Dispersive X-ray Fluorescence (EDXRF) and total reflection - X-ray absorption near-edge structure (TXRF-XANES) techniques. In our previous works, we successfully immobilized dithizone on PVC-based thin film substrates, creating novel Hg-selective membranes, and identified the optimal experimental parameters affecting mercury sorption from water samples. Nonetheless, the question remains, to what extent dithizone is responsible for mercury complexation on the membrane surface, or it just improves the membrane's active surface area thus simply improving the adsorptive effect. Using the EDXRF technique it appears that membranes functionalized with dithizone has a much higher efficiency (by up to threefold) in preconcetrating mercury from water matrices, compared to the non-functionalized membranes. Then, the membranes were also examined by means of TXRF-XANES and it was identified that indeed mercury-dithizone complex is produced on the membrane surface, which is responsible for the much higher mercury sorption, compared to the non-functionalized membranes. Also, the XANES mercury-dithizone spectrum is presented. Given polymer membrane simple manufacturing procedure and their low cost this study works towards establishing a new method for very low concentration mercury analysis (sub-ppb levels) as well as mercury collection from aqueous bodies, provided that the membranes are produced and used in large-scale routine works.

Keywords: Mercury (Hg), dithizone $\left(\mathrm{C}_{13} \mathrm{H}_{12} \mathrm{~N}_{4} \mathrm{~S}\right)$, polymer membranes, water matrices, heavy metal pollution.

\section{Introduction}

Mercury is considered a major environmental pollutant and one of the most toxic elements that may be found in aqueous bodies, since exposure to miniscule concentration $(\mathrm{ppb})$ can cause detrimental health effects (Elias et al., 2018; Kallithrakas-Kontos and Foteinis, 2016). It is a persistent and highly toxic heavy metal, which can cause severe biological toxicity on the nervous, digestive, and immune system, among others (Kallithrakas-Kontos and Foteinis, 2016).

Mercury occurs naturally (e.g. volcanic activity and weathering of rocks), but most of the mercury in the environment result from human activities; particularly from coal-fired power stations -the main source of mercury found in the atmosphere-, artisanal gold mining (where mercury is used to form an amalgam before being burnt off), landfilling, and dental materials (Riccardi et al., 2013; WHO, 2007). For example, only in the United States (US) around 44 tons of mercury are emitted annually from coal-fired power plants, accounting for more than $30 \%$ of emissions from all anthropogenic mercury sources (Liu et al., 2016).

Mercury largely occurs in its elemental $(\mathrm{Hg}(0)$ - a liquid or monatomic vapour), or its oxidized $\left(\mathrm{Hg}^{2+}\right.$ - divalent mercury or mercuric ion) form, with both undergoing redox changes in biotic and abiotic processes (Riccardi et al., 2013). Redox cycling between $\mathrm{Hg}(0)$ and $\mathrm{Hg}^{2+}$, which is driven by both photochemical processes and dark reactions, is the key mechanism for mercury transport in groundwater systems (Colombo et al., 2014). Furthermore, $\mathrm{Hg}^{2+}$ is the substrate for the production of neurotoxic methylmercury, which enters food webs and biomagnifies at higher trophic levels (Colombo et al., 2014). Therefore, even though $\mathrm{Hg}(0)$ is relatively unreactive, when oxidized to $\mathrm{Hg}^{2+}$ it becomes highly reactive, neurotoxic, nephrotoxic, hepatotoxic, and immunotoxic (Riccardi et al., 2013). 
As a result, mercury is classified a "Priority pollutant" in the EU (2000/60/EC), with an established limit for drinking water as low as $1 \mu \mathrm{g} \mathrm{L}^{-1} \mathrm{Hg}$ (Elias et al., 2018; Marguí et al., 2018). Furthermore, the World Health Organization (WHO) has recommended the reduction or elimination of releases of mercury and its compounds to the environment (WHO, 2007). Even though due to legislation initiatives mercury's industrial usage has been reduced (Hatzistavros and Kallithrakas-Kontos, 2014), the facts that: a) mercury has been used by humans for more than 3500 years (Kallithrakas-Kontos and Foteinis, 2016), and b) existing anthropogenic sources will continue to emit thousands of metric tonnes of mercury into the environment, adding to the naturally releases (e.g.volcanoes and deep sea vents) (Riccardi et al., 2013), are indicative of the extent of the problem. Thus, it is clear that mercury monitoring, assessment, and, most importantly, collection from natural water bodies remains imperative to protect human health and the environment.

Given mercury's high toxicity, various methods and technologies for its collection from the environment, such as phytoremediation, bioremediation, activated carbon adsorption, and extraction, have been proposed (Atwood and Zaman, 2006). The use of adsorptive matrices for mercury capture from aqueous streams has been extensively reported in literature, focusing on various thiol-functionalized materials like resins, particles, or composite materials as mercury ion capture modalities (Smuleac et al., 2005). Moreover, the use of chemical reagents, particularly thiol-based ligands, for mercury removal from aqueous systems have been proven effective in precipitating mercury (Atwood and Zaman, 2006). The reason is that, from the principles of hard-soft acid-base chemistry, mercury has a very strong affinity towards sulphur (Smuleac et al., 2005). Hence, $\mathrm{Hg}^{2+}$ is most frequently bonded to deprotonated thiol donors in natural organic matter and biological systems (Manceau et al., 2015).

Among the many thiol-based ligands that have been examined in the literature, e.g. see (Atwood and Zaman, 2006), dithizone is a well-established and -known ligand (e.g. see (Elly, 1973; Litman et al., 1977). In our previous work (Hatzistavros and Kallithrakas-Kontos, 2014), we created novel Hg-selective PVC-based thin film membranes and identified dithizone as a very promising ligand for mercury preconcentration on environmental water matrices, at trace (ppb) levels.

Here, we assess the effectiveness of the produced $\mathrm{Hg}^{2+}$ selective membranes, functionalized with dithizone, on mercury preconcentration/collection. To this end, we produced two types of membranes, one functionalized with dithizone and one without, and used them to collect mercury (25 ng $\mathrm{mL}-1)$ from water matrices. Measurements were carried out using EDXRF and XANES techniques. Furthermore, based on the preliminary results we discuss the possibility of the produced $\mathrm{Hg}^{2+}$ selective membranes to act as a low cost method for mercury monitoring in natural water bodies, and even mercury collection, should they being produced and employed at large scale.

\section{Materials and methods}

\subsection{Chemical reagents and solvents}

For membrane and solvent preparation the following, high quality, analytical reagents were used. For the membrane matrix high molecular weight polyvinyl chloride, i.e. PVC, was sourced from Fluka' ${ }^{\mathrm{TM}}$ (No. 81387). The membrane solution was prepared using: as plasticizer dibutyl phthalate (DBP) sourced from Riedel-de Haën (No. 36736, Pestanal $\left.{ }^{\circledR}\right)$; as ionophore, which is used to enhance anion binding on the membrane, 5,5-dithiobis(2nitrobenzoic acid) (DTNB) sourced from Fluka $^{\text {TM }}$ (No. 43760, Ellman's reagent); and as the complexing reagent (ligand) dithizone, sourced from Sigma-Aldrich ${ }^{\circledR}$ (No. 43820).

For the solvents preparation ultrapure water (ASTM Type I) was used, where the dithizone and mercury (Fluka Mercury Standard for Atomic Absorption Spectroscopy AAS (1000 $\pm 4 \mathrm{mg} \mathrm{L-1}$, Cat. No. 16482) were mixed. The above chemical reagents were in solid form and hence tetrahydrofuran (THF), sourced from Sigma-Aldrich ${ }^{\circledR}$ (No. 401757), was used for their dilution. Finally, as mentioned above, typical ultrapure water (ASTM Type I) was used throughout the work.

\subsection{Membrane and solvent preparation}

Membranes were prepared directly on thin films (Mylar ${ }^{\circledR}$ $2.5 \mu \mathrm{m})$, placed in cylindrical XRF sample plastic cups (Chemplex Cat. No. 1540) at the Analytical and Environmental Chemistry Laboratory, Technical University of Crete, Greece. Specifically, the Membrane solution was prepared by mixing the solvent (PVC matrix) with the plasticizer (dibutylphthalate), the complexing reagent, i.e. ligand (dithizone), and ionophore (DTNB). An in depth analysis and discussion on selective membrane preparation procedure can be found in our previous work (Hatzistavros and Kallithrakas-Kontos, 2014).

In total the performance of two membranes was examined herein. One prepared under the optimal conditions identified by (Hatzistavros and KallithrakasKontos, 2014), i.e. Mylar ${ }^{\circledR} 2.5 \mu \mathrm{m}$ was used as the thin film substrate and the membrane composition (w/w) was $52 \%$ PVC, 32\% dibutyl phthalate, 9.5\% dithizone and $6.5 \%$ ionophore, all diluted in THF solvent, and one prepared using the above mixture but without the ligand (dithizone). The latter membrane was prepared to identify the effect the complexing reagent, in this case dithizone, on the membrane efficiency and whether mercury ions would bind on cation selective membrane regardless the ligand. Once the membranes were prepared they were immersed, for $16 \mathrm{~h}$, on a large laboratory flask, containing $1000 \mathrm{~mL}$ ultrapure water and $25 \mathrm{ng}$ of mercury. The sample was stirred to enhance the mobility of the mercury ions. After $\mathrm{Hg}$ selective collection procedure both membranes were rinsed with high purity water and they were kept for analysis. 
For the TXRF-XANES analyses instead of the Mylar ${ }^{\circledast}$ $2.5 \mu \mathrm{m}$ film, the membranes were immobilization on quartz reflectors. Specifically, the membrane solution was placed on the center of the quartz glass, producing a $10-\mathrm{mm}$ diameter spot and was left to dry at room temperature (a detailed discussion of this procedure can be found in (Koulouridakis and Kallithrakas-Kontos, 2004)). Once the reflectors with each membrane, one with and one without dithizone, were dried they were immersed in the large laboratory flask ( $25 \mathrm{ng} \mathrm{L}^{-1}$ mercury). Also, in order to produce the XANES reference spectra for divalent mercury and for $\mathrm{Hg}^{2+}$-dithizone two solvents were prepared. The first one by diluting $25 \mathrm{ng}$ of mercury in $1000 \mathrm{~mL}$ ultrapure water (ASTM Type I), while in the second one apart from the $25 \mathrm{ng}$ of mercury also dithizone was added. Each solvent was stirred to allow the uniform distribution of its content. Then, $10 \mu \mathrm{L}$ were taken from each solvent and placed on a TXRF quartz reflector and it was left to dry (without heating).

\subsection{Apparatus}

Sample analyses were performed by means of an Energy Dispersive X-ray fluorescence (EDXRF) unit, to identify the $\mathrm{Hg}^{2+}$ content which is also crucial for the XANES measurements, and also by means of X-ray Absorption Near Edge Structure Spectroscopy (XANES) to identify if a mercury-dithizone complex is produced on the membrane surface.

\subsubsection{Energy dispersive $X$-ray (EDXRF) analyses}

$\mathrm{X}$-ray fluorescence (XRF) analysis is considered a rather simple analytical technique, in terms of its application and interpretation, offering qualitative and quantitative elemental information at trace level (Karydas et al., 2018). Here we used the Energy Dispersive X-ray Fluorescence (EDXRF) technique to estimate the $\mathrm{Hg}^{2+}$ concentration, at sub-ppb levels. Samples were measured by means of an AMETEK $^{\circledR}$ SPECTRO XEPOS unit, a widely used EDXRF spectrometer for environmental monitoring and assessment applications (Kallithrakas-Kontos et al., 2016). The unit has a 12-position autosampler and excitation is achieved through an air-cooled Palladium (Pd) anode $X$-ray end window tube, with maximum power and voltage $50 \mathrm{~W}$ and $50 \mathrm{kV}$, respectively (Hatzistavros and Kallithrakas-Kontos, 2014). Furthermore, the unit has three excitation modes, the Compton secondary/molybdenum, the Barkla Scatter/aluminum oxide, and the Bragg crystal/highly oriented pyrolytic graphite (HOPG). The unit operated with a silicon drift detector (SDD), with Peltier cooling (i.e. it operates at $-25 \circ \mathrm{C}$ without the need for liquid nitrogen input) and an $8 \mu \mathrm{m}$ Moxtek Dura-Be window. Finally, the peak to background ratio is $5000: 1$ and the detector resolution is $160 \mathrm{eV}$ at $5.9 \mathrm{keV}$ (Foteinis et al., 2013).

Here the $\mathrm{Hg}^{2+}$ measurements were performed using the Compton secondary/molybdenum mode at $40 \mathrm{kV}$ and $0.9 \mathrm{~mA}$. Measurements were performed using helium gas flushing, instead of in air atmosphere. Each sample was irradiated for $5 \mathrm{~min}(300 \mathrm{~s})$. After irradiation, the raw data were processed using X-Lab Pro 4.0 quantitation software and TurboQuant screening method. $\mathrm{Hg}^{2+}$ quantification was vital, not only to estimate the efficiency of each membrane in mercury sorption, but also and for XANES measurements, since the mercury content will determine the XANES irradiation time, among others.

\subsubsection{X-ray absorption near edge structure spectroscopy (XANES) analysis}

The effectiveness of the ligand under study, i.e. dithizone, was assessed by investigating the near-edge part of the absorption spectrum in the tender (X-ray absorption nearedge structure, XANES). In contrast to EDXRF, which can estimate the elemental composition down to the $\mathrm{ng} / \mathrm{g}$, XANES offers distinction of chemical forms selected/detected elements, i.e. speciation (Wrobel et al., 2016), in this case $\mathrm{Hg}^{2+}$ and $\mathrm{Hg}^{2+}$-dithizone complex. In general, XANES possesses a good chemical-state and ligand sensitivity, because of participation of unoccupied valence orbitals in the excited state and is also sensitive to distant atoms at low kinetic energy of the emitted photoelectron (Manceau et al., 2015). Furthermore, XANES reaches optimum sensitivity when the excitation geometry are carefully adjusted to maximize the fluorescence signal of the analyte element and to reduce spectral background (Wrobel et al., 2016). Nonetheless, the main hurdles for XANES measurements in identifying $\mathrm{Hg}$-binding environments include a) poor spectral resolution due to the large $\mathrm{Hg}$ core-hole lifetime broadening (up to $5.5 \mathrm{eV}$ at the L3-edge), which severely degrades chemical-state and ligand sensitivity; and b) the high difficulty to interpret XANES spectra in quantitative molecular structural terms (Manceau et al., 2015).

In this work, XANES measurements were performed at Elettra Sincrotrone Trieste (IAEA End-station). Specifically, the International Atomic Energy Agency (IAEA) through its program activities promotes and supports interdisciplinary research at synchrotron radiation facilities, including the Elettra Sincrotrone Trieste (EST), in Italy (Karydas et al., 2018). In particular, the Physics Section of the IAEA has developed and installed an experimental X-Ray Spectrometry facility, as end-station at the XRF beamline of EST, using $40 \%$ of the beam time. The beam is accessible to IEA end-users, such as this case, allowing the synergistic application of various variants of XRF techniques, including XANES (IAEA, 2018).

The ligand environment of the membranes used for the selective absorption of $\mathrm{Hg}^{2+}$ was studied by means of $\mathrm{Hg}$ L3 XANES analysis ( $\mathrm{Hg}$-L3 edge at 12,284 eV, using the 20-BM-B beamline (sector 20)). The XANES data obtained from the Elettra Sincrotrone Trieste (Figure 1) were processed by ATHENA tool, typically used for XANES data processing (Wrobel et al., 2016). The X-ray fluorescence beamline of the Elettra Sincrotrone Trieste is based on a $\mathrm{Si}(111)$ double crystal monochromator (resolving power of $1.4 \times 10^{-4}$, photon flux of $108-109 \mathrm{~s}^{-1}$ in the energy range between 3.65 and $14.5 \mathrm{keV}$ ). Moreover, IAES's advanced $\mathrm{X}$-ray spectrometry instrument is equipped with a multi-axis motorized sample manipulator (four linear and three rotational stages that provides different degrees of 
freedom to align the sample surface relative to the incident beam); an ultra-thin window (UTW) silicon drift detector (SDD, Bruker Nano GmbH, XFlash 5030, $30 \mathrm{~mm}^{2}$ nominal crystal area, $450 \mu \mathrm{m}$ crystal thickness and $131 \mathrm{eV}$ energy resolution at $\mathrm{Mn}-\mathrm{K} \alpha$ ) (Shaltout et al., 2018). As mentioned above, using the XANES spectra it is possible to identify the chemical speciation of trace elements on the sample surface (Karydas et al., 2018), i.e. on the TXRF quartz reflectors.

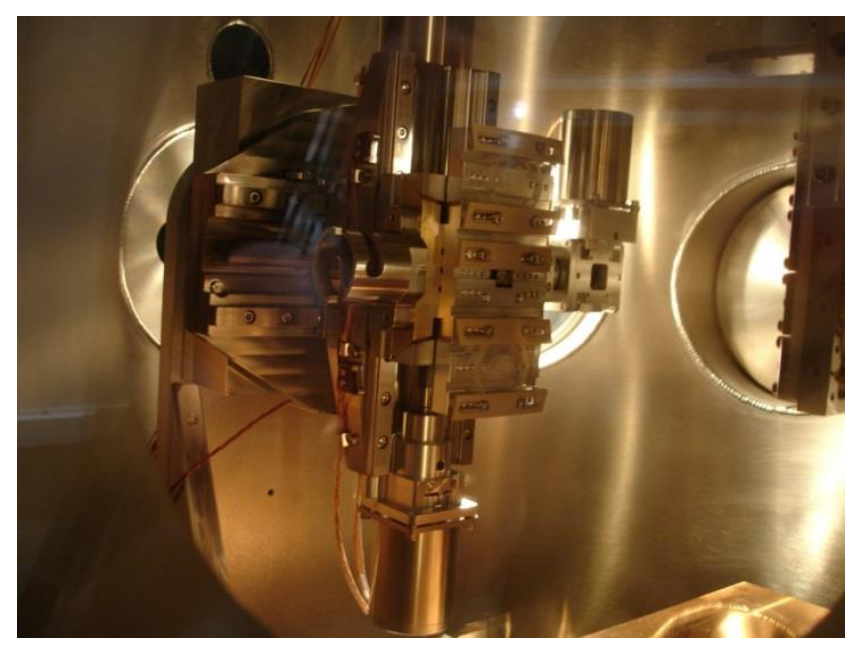

Figure 1. The quartz reflectors with the samples on their surfaces, at TXRF geometry inside the vacuum chamber for XANES measurements at the Elettra Sincrotrone Trieste, Italy

\section{Results and discussion}

\subsection{EDXRF results}

In our previous studies, PVC-based, cation selective membranes functionalized dithizone phthalate have been identified as an excellent preconcentration method for mercury analysis in water matrices, using the TXRF or the EDXRF technique (e.g. see (Hatzistavros and KallithrakasKontos, 2014; Koulouridakis and Kallithrakas-Kontos, 2004; Marguí et al., 2018). Here, we applied EDXRF technique to identify the effectiveness of the ligand, i.e. dithizone, on the proposed polymer membranes. Figure 2 shows EDXRF results for polymer membranes funtionalized with the selected ligand and a blank sample.

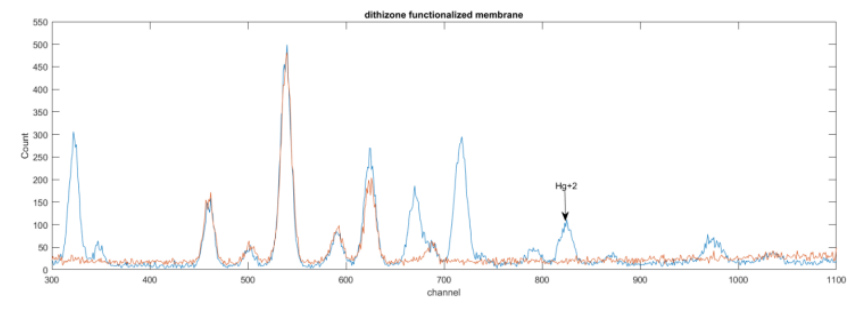

Figure 2. EDXR results for the PVC-based membrane functionalized with dithizone (blue colour) and a blank sample (orange colour)

As far as the efficiency in mercury sorption is concerned, it was found that the dithizone-functionalized membrane exhibited a much higher (up to threefold) efficiency in preconcentrating mercury (data not sown). This is in agreement with the results obtained from our previous work using TXRF (Koulouridakis and Kallithrakas-Kontos, 2004).

This suggest that the presence of a ligand, in this case dithizone a well-known mercury absorbent from water matrices, has a profound effect for mercury preconcertation. Results are also indicative of the many available possibilities of the produced PVC-based polymer membranes, which if applied at large-scale can offer a reliable, sensitive, rapid and most probably economic method, not only for mercury preconcetration, monitoring and assessment, but also for mercury removal from natural water bodies provided that they are used in large-scale routine works.

Even so, results are suggestive of the superiority of the membrane that is functionalized with dithizone, EDXRF cannot shed light if, and to what extent, a mercurydithizone complex is produced. For this reason the XANES technique was applied, as shown below.

\subsection{XANES results}

In Figure 3 the XANES spectra of the two membranes, i.e. with and without the ligand (dithizone), as well as the XANES spectra for $\mathrm{Hg}^{2+}$ and $\mathrm{Hg}^{2+}$-dithizone complex are shown. The reference XANES spectra for $\mathrm{Hg}^{2+}$ and $\mathrm{Hg}^{2+}-$ dithizone complexo were obtained by measuring the absorption of the model compounds (i.e. the reference solutions that were placed on TXRF quartz glasses). The $\mathrm{Hg}^{2+}$ XANES reference spectra appears to have an intense pre-edge peak, i.e. the energy position of the first peak (E1), an indicator of $\mathrm{Hg}$ oxidation, at about 12284.5 $\mathrm{eV}$, which is consistent with the literature (Colombo et al., 2014). The $\mathrm{Hg}^{2+}$-dithizone complex XANES reference spectra appears to have an E1 at 1290 e. As far as the dithizone-functionalized membrane is concerned it exhibited an E1 of $1289.5 \mathrm{eV}$, very similar to the $\mathrm{Hg}^{2+}$-dithizone complex XANES reference spectra. On the other hand, the non-fuctionalized membrane intense pre-edge peak is not very clear, but it appears to match the $\mathrm{Hg}^{2+}$ XANES reference spectra. It has to be noted that the membranes XANES spectra had an overall low quality, especially those the non-functionalized membrane, which is attributed to the very low concentrations of $\mathrm{Hg}^{2+}$ on their surfaces. This was expected, since main target of the produced membranes is mercury preconcertation and not collection, while the non functionalized membrane had a much lower efficiency in mercury sorption, which is clearly reflected in the XANES spectra low quality.

Overall, results are suggestive of the non-fuctionalized membrane had indeed adsorbed $\mathrm{Hg}^{2+}$, but at a very low concertation as identified by the EDXRF technique, which is depicted in the XANES spectra low quality. On the other hand, the XANES spectra quality of the dithizonefunctionalized membrane was much better, since it the mercury sorption was much higher, but most importantly the spectra fitted the XANES spectra of the reference $\mathrm{Hg}^{2+}$-dithizone complex. Therefore, it is shown that mercury is complexed on the membrane surface due to 
the presence of dithizone, and not that dithizone just improved the membrane's surface area thus simply improving mercury's adsorptive effect.

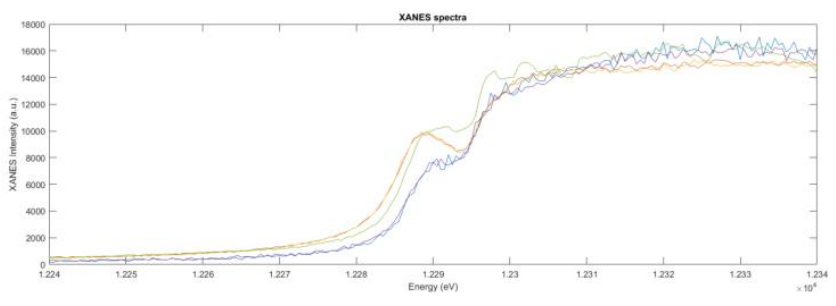

Figure 3. TXRF-XANES spectra of $\mathrm{Hg}^{2+}$ (red and orange) and $\mathrm{Hg}^{2+}$-dithizone complex (purple) references along with the XANES spectra of dithizone-functionalized membrane (blue) and the non-functionalized membrane (green)

Given the polymer membrane simple manufacturing procedure, they can be produced without the need of specialized equipment of personnel, and their overall low cost, the chemical reagents required are easy to find and not expensive given the intended purpose, the many possibilities of the cation selective membranes emerge. Specifically, the membranes can be effectively used for mercury preconcetration from natural water matrices, such as seawater. This is imperative since heavy metals pollution in seawater has been extensively reported (Chatzisymeon, 2016)) and given mercury's high toxicity at miniscule concetration (ng $\mathrm{L}^{-1}$ ), its effective monitoring and assessment remains a difficult task. Furthermore, another possibility that could be addressed in future works is the use of the dithizone-functionalized polymer membranes for large-scale mercury collection from natural waterbodies as well as from industrial waterborne and possibly from airborne emissions, such as from coalfired power stations emissions, provided that the membranes are produced and used in large-scale routine works.

\section{Conclusions}

This study worked towards establishing a new method for very low concentration mercury analysis (sub-ppb levels), by examining the mercury species and the complexes that are formed in PVC-based $\mathrm{Hg}^{2+}$-selective membranes, futionalized with dithizone. Using the EDXRF technique it was found that the dithizone-functionalized membrane efficiency in mercury sorption is up to threefold higher, compared to non-functionalized membranes. This is in agreement with results obtained from our previous work using TXRF. In order to identify if mercury is complexed with dithizone on the membrane surface or if dithizone just improves the membrane's active surface area thus simply improving mercury's adsorptive effect the XANES technique was applied. Furthermore, the XANES mercurydithizone spectrum is given.

It was found that mercury complexes with dithizone directly on the membranes surface, thus substantially improving the membrane's efficiency in preconcentrating mercury from natural water matrices. Also, Then, the membranes were also examined by means of and it was identified that indeed mercury-dithizone complex is produced on the membrane surface, which is responsible for the much higher mercury sorption, compared to the non-functionalized membranes. Given the simple manufacturing procedure, even by non-specialized personnel, and their overall low cost the polymer membranes are excellent tool for mercury preconcetration at trace level (ng L ${ }^{-1}$ ) and subsequently for their use as a robust tool for monitoring and assessment application in natural water bodies. Future applications could also include the large-scale mercury collection not only from natural waterbodies, but also from industrial waterborne and possibly from airborne emissions, such as from coal-fired power stations emissions, provided that the membranes are produced and used in large-scale routine works.

\section{Acknowledgements}

We would like to thank the International Atomic Energy Agency (IAEA) for providing funding through the coordinated research project G42005, entitled "Experiments with Synchrotron Radiation for Modern Environmental and Industrial Applications", by the Research Contract No. 18262. We would like also to thank Dr Alessandro Migliori for helping with the XANES measurements. Finally, we greatly appreciate the beamtime awarded by IAEA and the Elettra Sincrotrone Trieste.

\section{References}

Atwood D.A. and Zaman M.K. (2006), Mercury Removal from Water, in Recent Developments in Mercury Science, Atwood D.A. (Ed.), Springer Berlin Heidelberg, Berlin, Heidelberg, pp. 163-182.

Chatzisymeon E. (2016), Inactivation of bacteria in seafood processing water by means of UV treatment. Journal of Food Engineering, 173, 1-7.

Colombo M.J., Ha J., Reinfelder J.R., Barkay T. and Yee N. (2014), Oxidation of $\mathrm{Hg}(0)$ to $\mathrm{Hg}(\mathrm{II})$ by diverse anaerobic bacteria. Chemical Geology, 363, 334-340.

Elias G., Margui E., Diez S. and Fontas C. (2018), Polymer Inclusion Membrane as an Effective Sorbent To Facilitate Mercury Storage and Detection by $\mathrm{X}$-ray Fluorescence in Natural Waters. Analytical Chemistry, 90, 4756-4763.

Elly C.T. (1973), Dithizone Procedure for Mercury Analysis. Journal (Water Pollution Control Federation), 45, 940-945.

Foteinis S., Kallithrakas-Kontos N.G. and Synolakis C. (2013), Heavy Metal Distribution in Opportunistic Beach Nourishment: A Case Study in Greece. The Scientific World Journal, 5.

Hatzistavros V.S. and Kallithrakas-Kontos N.G. (2014), X-ray fluorescence mercury determination using cation selective membranes at sub-ppb levels. Analytica Chimica Acta, 809, $25-29$.

IAEA (2018), Experiments with Synchrotron Radiation for Modern Environmental and Industrial Applications - Project Code G42005. IAEA, Participating Countries: Australia, Croatia, Germany, Greece, Indonesia, Italy, Jordan, Slovenia, Spain, Thailand, United Arab Emirates, United States of America.

Kallithrakas-Kontos N. and Foteinis S. (2016), Recent Advances in the Analysis of Mercury in Water - Review. Current Analytical Chemistry, 12. 
Kallithrakas-Kontos N., Foteinis S., Paigniotaki K. and Papadogiannakis M. (2016), A robust X-ray fluorescence technique for multielemental analysis of solid samples. Environmental Monitoring and Assessment, 188, 120.

Karydas A.G., Czyzycki M., Leani J.J., Migliori A., Osan J., Bogovac M., Wrobel P., Vakula N., Padilla-Alvarez R., Menk R.H., Gol M.G., Antonelli M., Tiwari M.K., Caliri C., Vogel-Mikuš K., Darby I. and Kaiser R.B. (2018), An IAEA multi-technique Xray spectrometry endstation at Elettra Sincrotrone Trieste: benchmarking results and interdisciplinary applications. Journal of Synchrotron Radiation, 25, 189-203.

Koulouridakis P.E. and Kallithrakas-Kontos N.G. (2004), Selective Mercury Determination after Membrane Complexation and Total Reflection X-ray Fluorescence Analysis. Analytical Chemistry, 76, 4315-4319.

Litman R., Williams E.T. and Finston H.L. (1977), Extraction of mercury by dithizone and stability of the extracted complex. Analytical Chemistry, 49, 983-987.

Liu Z., Li C., Sriram V., Lee J.-Y. and Brewe D. (2016), XANES study of elemental mercury oxidation over RuO2/TiO2 and selective catalytic reduction catalysts for mercury emissions control. Fuel Processing Technology, 153, 156-162.

Manceau A., Lemouchi C., Rovezzi M., Lanson M., Glatzel P., Nagy K.L., Gautier-Luneau I., Joly Y. and Enescu M. (2015), Structure, Bonding, and Stability of Mercury Complexes with Thiolate and Thioether Ligands from High-Resolution XANES Spectroscopy and First-Principles Calculations. Inorganic Chemistry, 54, 11776-11791.

Marguí E., Queralt I., Guerra M. and Kallithrakas-Kontos N. (2018), Mercury determination at trace levels using membrane preconcentration and benchtop total reflection X-ray fluorescence analysis. Spectrochimica Acta Part B: Atomic Spectroscopy, 149, 84-90.

Riccardi D., Guo H.-B., Parks J.M., Gu B., Summers A.O., Miller S.M., Liang L. and Smith J.C. (2013), Why Mercury Prefers Soft Ligands. The Journal of Physical Chemistry Letters, 4, 2317-2322.

Shaltout A.A., Harfouche M., Ahmed S.I., Czyzycki M. and Karydas A.G. (2018), Synchrotron radiation total reflection Xray fluorescence (SR-TXRF) and X-ray absorption near edge structure (XANES) of fractionated air particulates collected from Jeddah, Saudi Arabia. Microchemical Journal, 137, 7884.

Smuleac V., Butterfield D.A., Sikdar S.K., Varma R.S. and Bhattacharyya D. (2005), Polythiol-functionalized alumina membranes for mercury capture. Journal of Membrane Science, 251, 169-178.

WHO (2007), Exposure to Mercury: A Major Public Health Concern, Preventing disease through healthy environments.

Wrobel P.M., Bogovac M., Sghaier H., Leani J.J., Migliori A., Padilla-Alvarez R., Czyzycki M., Osan J., Kaiser R.B. and Karydas A.G. (2016), LabVIEW interface with Tango control system for a multi-technique $\mathrm{X}$-ray spectrometry IAEA beamline end-station at Elettra Sincrotrone Trieste. Nuclear Instruments and Methods in Physics Research Section A: Accelerators, Spectrometers, Detectors and Associated Equipment, 833, 105-109. 